\title{
A study the effect of supplementation vitamin $C$ and dietary calcium on: I- Some productive performance in two different strains of broiler chicks under heat stress conditions
}

\author{
A. L. D. AL- Khauzai K.N.Tahir A.S.M* AL- Tepery \\ Coll. of Vet.Med./ Univ.of AL-Qadissiyah
}

\begin{abstract}
This study was undertaken to evaluate the effect of adding vitamin $\mathrm{C}$ at $(0,150 \mathrm{mg} / \mathrm{kg}$ feed) and dietary calcium at $(0.9,1.1 \%)$ on some productive performance of two different strains of broiler (Ross and Hubbard).A total of one hundred ninety two birds(96 chicks for each strain) one day old unsexed were used in this study. Each strain distributed randomly to four groups (24) chicks for each treatment, two replicates of (12)chicks for each.First group was fed on basal diet supplemented with $(0.0$ vitamin $\mathrm{C}+0.9 \%$ calcium $)$.Second group was fed on the same basal diet supplemented with ( 0.0 vitamin $\mathrm{C}+1.1 \%$ calcium).Third group was fed on basal diet supplemented with (150 $\mathrm{mg}$ vitamin $\mathrm{C} / \mathrm{kg}$ feed $+0.9 \%$ calcium) and fourth group was fed on basal diet supplemented with $(150 \mathrm{mg}$ vitamin $\mathrm{C} / \mathrm{kg}$ feed $+1.1 \%$ calcium).The results of this study showed that growth performance of chicks was significantly improved in Co-supplemented vitamin $\mathrm{C}$ and calcium $(1.1 \%)$ and there was no significant interaction between vitamin $\mathrm{C}$ supplementation and calcium on dressing percentage and mortality rate.
\end{abstract}

\section{Introduction}

It is well known that high ambient temperature coupled with high humidity has a detrimental effect on the poultry industry by decreasing performance such as (body weight, weight gain. feed intake and feed conversion ratio(FCR).as well as profitability of broiler production, physiologically heat stress causes the release of corticosterone and catecholamines and initiates lipid peroxidation in cell membranes (1، 2). There are numerous methods to alleviate the negative effects of high temperature on performance of poultry; such methods are focused mostly on dietary manipulation. For this purpose vitamin $\mathrm{C}$ was used in poultry diets, because of their anti-stress effects and also because of their synthesis was reduced during heat stress $(3,1)$.In the same way under heat stress condition, birds are not able to synthesize sufficient amount of vitamin C (4).Positive effect of vitamin $\mathrm{C}$ supplementation in broiler chickens have been documented previously (5 and 6). (7) noted that during summer months, broiler performance was decreased and increased in mortality rate. (8) found that ascorbic acid supplementation in broiler diet reduced mortality by $14.6 \%$ during heat stress. Calcium play two an important physiological roles in the avian subject, first a provides the structural strength of avian skeleton by formation of calcium salt, second it plays vital role in many biochemical reaction with body via its concentration in the intracellular fluid (9).Vitamin C nutrition should have influence on calcium metabolism in young chicks which posses no ability to synthesize vitamin C (10).(5) stated that metabolism of calcium may also be influenced by dietary vitamin $\mathrm{C}$, and the binding capacity of calcium binding protein is significantly improved with vitamin $\mathrm{C}$ supplementation .The present study was carried out to determine the effect of vitamin $\mathrm{C}$ and calcium supplementation on some productive performance for broilers under heat stress conditions.

\footnotetext{
* Part of M.sc. Thesis for the third researcher .
} 


\section{Materials and Methods}

The experiment was conducted in commercial broiler farm with two different strains of broiler chicks, (Ross and Hbbard Flex). A total of one hundred ninety two one day old unsexed broiler chicks (96 chicks for each strain) were used in this study and were randomly distributed into four floor pens per each (24 chicks) with two replicates consisting (12) chicks for each. The chicks were reared in completely enclosed house and exposed to continuous heat stress. The temperature in the heat stress was increase in three phases $\left(22,28,33 \mathrm{C}^{\circ}\right)$. Feed and water were given ad Libitum, and the chicks vaccinated against New castle and Gumboro diseases similar management conditions were maintained for all group through out the experiment period.The experiment was carried out from September $15^{\text {th }}$ to October $20^{\text {th }} 2006$.

Table (1):A summary of dietary supplementation of the experimental treatments

\begin{tabular}{|c|c|l|l|}
\hline Treatment-1- & \multicolumn{1}{|c|}{ Treatment -2- } & \multicolumn{1}{|c|}{ Treatment -3- } & \multicolumn{1}{c|}{ Treatment -4- } \\
\hline $\begin{array}{l}\text { Basal diet }+0.0 \\
\text { vitamin C+0.9\% ca }\end{array}$ & $\begin{array}{l}\text { Basal diet }+0.0 \\
\text { vitamin C+1.1\% ca }\end{array}$ & $\begin{array}{l}\text { Basal diet }+150 \mathrm{mg} \\
\text { Vitamin C/kg feed } \\
+0.9 \% \mathrm{ca}\end{array}$ & $\begin{array}{l}\text { Basal diet +150 mg } \\
\text { vitaminC/kgfeed+1.1 } \\
\% \mathrm{ca}\end{array}$ \\
\hline
\end{tabular}

The basal diet was formulated according to (11). The ingredients and chemical composition of the basal diet are shown in Table (2).

Table (2) Ingredients and chemical composition of experimental diet .

\begin{tabular}{|l|l|l|}
\hline \multicolumn{1}{|c|}{ Ingredients } & \multicolumn{1}{c|}{$\%$} & \multicolumn{1}{c|}{$\%$} \\
\hline Yellow corn & 35.5 & 35.0 \\
Wheat & 23.5 & 23.5 \\
Wheat bran & 4.0 & 4.0 \\
Fish meal & 5.0 & 5.0 \\
Soybean meal (44\%) & 30.0 & 30.0 \\
Lime stone & 0.5 & 1.0 \\
Di calcium phosphate & 1.0 & 1.0 \\
Minerals and vitamins premix & 0.2 & 0.2 \\
Lysine & 0.2 & 0.2 \\
methionine & 0.1 & 0.1 \\
\hline Total & 100.00 & 100.00 \\
\hline Chemical composition calculated(\%) & & \\
\hline Metabolisable Energy (kcal/kg) & 2918 & 2901 \\
Crude protein(cp)\% & 23.3 & 23.2 \\
Calcium(ca)\% & 0.9 & 1.1 \\
Phosphorus(p)\% & 0.82 & 0.81 \\
Available (p)\% & 0.57 & 0.56 \\
Ca :p & $1: 1.6$ & $1: 2$ \\
\hline
\end{tabular}

\section{Measurements :-}

Body weight, weight gain and feed consumption were recorded weekly during the experimental period. Mortality was checked on weekly basis. Feed conversion ratio (FCR)calculated as the ratio of feed intake during a particular week to the weight gain during the same week. At the end of experiment, three chicks were selected from each treatment group, weighed and slaughtered to determine the dressing percentage after removing of head, shank and alimentary tract. 


\section{Results and discussion}

\section{Growth performance \\ Body weight:-}

Results of the present study showed that a dietary supplement of vitamin $\mathrm{C}$ at $150 \mathrm{mg} / \mathrm{kg}$ feed as combination with $1.1 \%$ ca, had the heaviest body weight as compared with other treatment groups (Table3) such combination can offer potential protective management practice in preventing heat stress related to losses in performance of broiler chicks exposed to heat stress. Similar results were obtained by (12).

\section{Weight gain:-}

Supplemental vitamin C coupled with calcium significantly $(\mathrm{P}<0.01)$ increased weight gain, the highest weight gain was in chicks fed $150 \mathrm{mg}$ vitamin C $/ \mathrm{kg}$ feed with $1.1 \%$ ca (Table 4). (13) found that vitamin $\mathrm{C}$ supplementation increased weight gain. (14) stated that greater weight gain found in chicks fed supplement vitamin $\mathrm{C}$.

\section{Feed consumption}

The effect of supplemental dietary vitamin $\mathrm{C}$ and calcium during heat stress of broiler chicks are shown in (Table 5). Supplemental vitamin C significantly increased feed intake $(\mathrm{P}<0.01)$ and also fed $1.1 \%$ calcium affected significantly $(\mathrm{P}<0.01)$ on feed intake as compared with
$0.9 \%$ ca. (15) noted that vitamin C supplementation in feed at $200 \mathrm{mg} / \mathrm{kg}$ during heat stress increased feed intake of birds. Similar results were obtained previously $(12,16)$.

\section{Feed conversion ratio (FCR):}

Table(5) showed that dietary supplementation of vitamin C $(150 \mathrm{mg} / \mathrm{kg}$ feed) and $0.9 \%$ calcium improved the (FCR). (16) have demonstrated that (FCR) was significantly improved in broilers fed with vitamin $C$. (17) reported that an improved feed conversion ratio by Ascorbic acid supplementation to Japanese quail reared under high temperature. (12) also detected an improvement in feed conversion ratio of broilers as result of vitamin $\mathrm{C}$ supplementation during heat stress. On the other hand, (18). did not find improved in broiler chicks

\section{Mortality rate and dressing percentage:-}

The results presented in Table (6) showed that mortality rate of birds during the experimental period were not influenced by dietary supplementation of vitamin $C$, similar results were obtained by (5).Dressing percentage also did not differ significantly when interaction between vitamin $\mathrm{C}$ and calcium were done, this study coincided with the finding of (19). 
Table(3): The effect of dietary supplementation vitamin C and calcium on body weight

\begin{tabular}{|c|c|c|c|c|c|c|c|c|}
\hline Treatment & \multicolumn{4}{|c|}{$0.0 \mathrm{mg}$ Vit.C/kg feed } & \multicolumn{4}{|c|}{$150 \mathrm{mg}$ Vit.C/kg feed } \\
\hline & \multicolumn{2}{|c|}{$0.9 \% \mathrm{Ca}$} & \multicolumn{2}{|c|}{$1.1 \% \mathrm{Ca}$} & \multicolumn{2}{|c|}{$0.9 \% \mathrm{Ca}$} & \multicolumn{2}{|c|}{$1.1 \% \mathrm{Ca}$} \\
\hline & Hubbard & Ross & Hubbard & Ross & Hubbard & Ross & Hubbard & Ross \\
\hline Age(week) & Mean \pm SE & Mean \pm SE & Mean \pm SE & Mean \pm SE & Mean \pm SE & Mean \pm SE & Mean \pm SE & Mean \pm SE \\
\hline 1 & $\begin{array}{c}\mathrm{A} \\
124.500 \pm 0.835\end{array}$ & $\begin{array}{c}\mathrm{B} \\
138.500 \pm 0.835\end{array}$ & $\begin{array}{c}\text { B } \\
142.000 \pm 0.835\end{array}$ & $\begin{array}{c}\text { BCG } \\
149.000 \pm 0.835\end{array}$ & $\begin{array}{c}\text { BCDG } \\
137.000 \pm 0.835\end{array}$ & $\begin{array}{c}\text { BCEG } \\
147.000 \pm 0.835\end{array}$ & $\begin{array}{c}\text { BCEFG } \\
124.000 \pm 0.835\end{array}$ & $\begin{array}{c}\mathrm{G} \\
152.000 \pm 0.835\end{array}$ \\
\hline 2 & $\begin{array}{c}\mathrm{A} \\
251.100 \pm 0.909\end{array}$ & $\begin{array}{c}\mathrm{AB} \\
263.600 \pm 0.909\end{array}$ & $\begin{array}{c}\mathrm{A} \\
268.500 \pm 0.909\end{array}$ & $\begin{array}{c}\text { B } \\
296.400 \pm 0.909\end{array}$ & $\begin{array}{c}\mathrm{B} \\
249.900 \pm 0.909\end{array}$ & $\begin{array}{c}\mathrm{C} \\
270.000 \pm 0.909\end{array}$ & $\begin{array}{c}\mathrm{C} \\
281.000 \pm 0.909\end{array}$ & $\begin{array}{c}\mathrm{C} \\
302.000 \pm 0.909\end{array}$ \\
\hline 3 & $\begin{array}{c}\mathrm{A} \\
472.6 \pm 1.172\end{array}$ & $\begin{array}{c}\mathrm{A} \\
475.400 \pm 1.172\end{array}$ & $\begin{array}{c}\mathrm{AB} \\
481.000 \pm 1.172\end{array}$ & $\begin{array}{c}\mathrm{B} \\
527.500 \pm 1.172\end{array}$ & $\begin{array}{c}\mathrm{AB} \\
487.300 \pm 1.172\end{array}$ & $\begin{array}{c}\mathrm{C} \\
491.000 \pm 1.172\end{array}$ & $\begin{array}{c}\mathrm{B} \\
528.200 \pm 1.172\end{array}$ & $\begin{array}{c}\mathrm{B} \\
521.700 \pm 1.172\end{array}$ \\
\hline 4 & $\begin{array}{c}\mathrm{A} \\
673.400 \pm 0.838\end{array}$ & $\begin{array}{c}\text { B } \\
685.200 \pm 0.838\end{array}$ & $\begin{array}{c}\mathrm{C} \\
680.300 \pm 0.838\end{array}$ & $\begin{array}{c}\mathrm{A} \\
704.000 \pm 0.838\end{array}$ & $\begin{array}{c}\mathrm{A} \\
691.800 \pm 0.838\end{array}$ & $\begin{array}{c}\mathrm{C} \\
675.500 \pm 0.838\end{array}$ & $\begin{array}{c}\mathrm{C} \\
705.900 \pm 0.838\end{array}$ & D $727.6 \pm 0.838$ \\
\hline 5 & $\begin{array}{c}\mathrm{A} \\
942.300 \pm 1.711\end{array}$ & $\begin{array}{c}\mathrm{A} \\
965.100 \pm 1.711\end{array}$ & $\begin{array}{c}\mathrm{BC} \\
903.900 \pm 1.711\end{array}$ & $\begin{array}{c}\mathrm{A} \\
950.250 \pm 1.711\end{array}$ & $\begin{array}{c}\mathrm{BC} \\
922.200 \pm 1.711\end{array}$ & $\begin{array}{c}\mathrm{A} \\
940.300 \pm 1.711\end{array}$ & $\begin{array}{c}\mathrm{BC} \\
890.900 \pm 1.711\end{array}$ & $\begin{array}{c}\mathrm{B} \\
885.200 \pm 1.711\end{array}$ \\
\hline
\end{tabular}

Means with in different columns with different superscripts differ significantly $(\mathrm{P}<0.01)$. 
Table (4): The effect of dietary supplementation vitamin $\mathrm{C}$ and calcium on weekly weight gain.

\begin{tabular}{|c|c|c|c|c|c|c|c|c|}
\hline $\mathrm{T}_{1}$ & \multicolumn{4}{|c|}{$0.0 \mathrm{mg}$ Vit.C/kg feed } & \multicolumn{4}{|c|}{$150 \mathrm{mg}$ Vit.C/kg feed } \\
\hline & \multicolumn{2}{|c|}{$0.9 \% \mathrm{Ca}$} & \multicolumn{2}{|c|}{$1.1 \% \mathrm{Ca}$} & \multicolumn{2}{|c|}{$0.9 \% \mathrm{Ca}$} & \multicolumn{2}{|c|}{$1.1 \% \mathrm{Ca}$} \\
\hline & Hubbard & Ross & Hubbard & Ross & Hubbard & Ross & Hubbard & Ross \\
\hline Age(week) & Mean \pm SE & Mean \pm SE & Mean \pm SE & Mean \pm SE & Mean \pm SE & Mean \pm SE & Mean \pm SE & Mean \pm SE \\
\hline 1 & $\begin{array}{c}\mathrm{A} \\
85.500 \pm 1.356\end{array}$ & $\begin{array}{c}\mathrm{B} \\
97.500 \pm 1.356\end{array}$ & $\begin{array}{c}\mathrm{B} \\
103.000 \pm 1.356\end{array}$ & $\begin{array}{c}\mathrm{BC} \\
108.000 \pm 1.256\end{array}$ & $\begin{array}{c}\mathrm{BC} \\
97.000 \pm 1.356\end{array}$ & $\begin{array}{c}\mathrm{C} \\
105.000 \pm 1.356\end{array}$ & $\begin{array}{c}\mathrm{BC} \\
100.000 \pm 1.356\end{array}$ & $\begin{array}{c}\mathrm{C} \\
109.000 \pm 1.356\end{array}$ \\
\hline 2 & $\begin{array}{c}\mathrm{AB} \\
126.600 \pm 1.167\end{array}$ & $\begin{array}{c}\mathrm{A} \\
125.100 \pm 1.167\end{array}$ & $\begin{array}{c}\mathrm{B} \\
126.50 \pm 1.167\end{array}$ & $\begin{array}{c}\mathrm{AB} \\
147.400 \pm 1.167\end{array}$ & $\begin{array}{c}\mathrm{A} \\
112.900 \pm 1.167\end{array}$ & $\begin{array}{c}\mathrm{CD} \\
123.000 \pm 1.167\end{array}$ & $\begin{array}{c}\mathrm{CD} \\
139.000 \pm 1.167\end{array}$ & $\begin{array}{c}\mathrm{D} \\
150.000 \pm 1.167\end{array}$ \\
\hline 3 & $\begin{array}{c}\mathrm{AB} \\
221.500 \pm 1.588\end{array}$ & $\begin{array}{c}\mathrm{A} \\
211.800 \pm 1.588\end{array}$ & $\begin{array}{c}\mathrm{B} \\
212.500 \pm 1.588\end{array}$ & $\begin{array}{c}\mathrm{AB} \\
231.100 \pm 1.588\end{array}$ & $\begin{array}{c}\mathrm{A} \\
237.400 \pm 1.588\end{array}$ & $\begin{array}{c}\mathrm{BC} \\
221.100 \pm 1.588\end{array}$ & $\begin{array}{c}\mathrm{D} \\
247.200 \pm 1.588\end{array}$ & $\begin{array}{c}\mathrm{AB} \\
219.700 \pm 1.588\end{array}$ \\
\hline 4 & $\begin{array}{c}\mathrm{A} \\
200.800 \pm 1.296\end{array}$ & $\begin{array}{c}\mathrm{A} \\
209.800 \pm 1.296\end{array}$ & $\begin{array}{c}\mathrm{A} \\
199.300 \pm 1.296\end{array}$ & $\begin{array}{c}\mathrm{B} \\
176.500 \pm 1.296\end{array}$ & $\begin{array}{c}\mathrm{A} \\
204.500 \pm 1.296\end{array}$ & $\begin{array}{c}\mathrm{BC} \\
184.500 \pm 1.296\end{array}$ & $\begin{array}{c}\mathrm{BC} \\
177.700 \pm 1.296\end{array}$ & $\begin{array}{c}\mathrm{A} \\
205.900 \pm 1.296\end{array}$ \\
\hline 5 & $\begin{array}{c}\mathrm{A} \\
268.900 \pm 1.862\end{array}$ & $\begin{array}{c}\mathrm{A} \\
279.900 \pm 1.862\end{array}$ & $\begin{array}{c}\mathrm{C} \\
223.600 \pm 1.862\end{array}$ & $\begin{array}{c}\mathrm{B} \\
246.250 \pm 1.862\end{array}$ & $\begin{array}{c}\mathrm{C} \\
230.400 \pm 1.862\end{array}$ & $\begin{array}{c}\mathrm{A} \\
264.800 \pm 1.862\end{array}$ & $\begin{array}{c}\mathrm{B} \\
185.000 \pm 1.862\end{array}$ & $\begin{array}{c}\mathrm{B} \\
157.600-1.862\end{array}$ \\
\hline
\end{tabular}

Means with in different columns with different superscripts differ significantly $(\mathrm{P}<0.01)$. 
Table (5): The effect of dietary supplementation vitamin C and calcium on weekly feed consumption.

\begin{tabular}{|c|c|c|c|c|c|c|c|c|}
\hline \multirow{4}{*}{ Age(week) } & \multicolumn{4}{|c|}{$0.0 \mathrm{mg}$ Vit.C/kg feed } & \multicolumn{4}{|c|}{$150 \mathrm{mg}$ Vit.C/kg feed } \\
\hline & \multicolumn{2}{|c|}{$0.9 \% \mathrm{Ca}$} & \multicolumn{2}{|c|}{$1.1 \% \mathrm{Ca}$} & \multicolumn{2}{|c|}{$0.9 \% \mathrm{Ca}$} & \multicolumn{2}{|c|}{$1.1 \% \mathrm{Ca}$} \\
\hline & Hubbard & Ross & Hubbard & Ross & Hubbard & Ross & Hubbard & Ross \\
\hline & Mean \pm SE & Mean \pm SE & Mean \pm SE & Mean \pm SE & Mean \pm SE & Mean \pm SE & Mean \pm SE & Mean \pm SE \\
\hline 1 & $\begin{array}{c}\mathrm{a} \\
94.360 \pm 0.369\end{array}$ & $\begin{array}{c}\mathrm{a} \\
95.830 \pm 0.369\end{array}$ & $\begin{array}{c}\mathrm{a} \\
98.490 \pm 0.369\end{array}$ & $\begin{array}{c}\mathrm{a} \\
98.770 \pm 0.369\end{array}$ & $\begin{array}{c}\mathrm{a} \\
98.850 \pm 0.369\end{array}$ & $\begin{array}{c}\mathrm{a} \\
98.280 \pm 0.369\end{array}$ & $\begin{array}{c}\mathrm{a} \\
99.190 \pm 0.369\end{array}$ & $\begin{array}{c}\mathrm{a} \\
100.030 \pm 0.369\end{array}$ \\
\hline 2 & $\begin{array}{c}\mathrm{ABa} \\
178.290 \pm 0.880\end{array}$ & $\begin{array}{c}\mathrm{ABa} \\
184.565 \pm 0.880\end{array}$ & $\begin{array}{c}\mathrm{ABa} \\
184.590 \pm 0.880\end{array}$ & $\begin{array}{c}\mathrm{ABa} \\
188.470 \pm 0.880\end{array}$ & $\begin{array}{c}\mathrm{Ab} \\
185.080 \pm 0.880\end{array}$ & $\begin{array}{c}\mathrm{B} \\
187.460 \pm 0.880\end{array}$ & $\begin{array}{c}\mathrm{ABa} \\
187.530 \pm 0.880\end{array}$ & $\begin{array}{c}\mathrm{ABa} \\
190.400 \pm 0.880\end{array}$ \\
\hline 3 & $\begin{array}{c}\mathrm{a} \\
298.550 \pm 1.709\end{array}$ & $\begin{array}{c}\mathrm{a} \\
327.110 \pm 1.709\end{array}$ & $\begin{array}{c}\mathrm{a} \\
311.220 \pm 1.709\end{array}$ & $\begin{array}{c}\mathrm{A} \\
328.720 \pm 1.709\end{array}$ & $\begin{array}{c}\mathrm{a} \\
325.670 \pm 1.709\end{array}$ & $\begin{array}{c}\mathrm{a} \\
338.940 \pm 1.709\end{array}$ & a $328.690 \pm 1.709$ & $\begin{array}{c}\mathrm{a} \\
354.600 \pm 1.709\end{array}$ \\
\hline 4 & $\begin{array}{c}\mathrm{A} \\
390.630 \pm 0.699\end{array}$ & $\begin{array}{c}\mathrm{B} \\
402.010 \pm 0.669\end{array}$ & $\begin{array}{c}\mathrm{C} \\
399.980 \pm 0.699\end{array}$ & $\begin{array}{c}C \mathrm{C} \\
416.500 \pm 0.699\end{array}$ & $\begin{array}{c}\mathrm{AC} \\
414.330 \pm 0.699\end{array}$ & $\begin{array}{c}\text { B } \\
441.840 \pm 0.699\end{array}$ & $\begin{array}{c}\mathrm{AC} \\
426.090 \pm 0.699\end{array}$ & $\begin{array}{c}C \mathrm{C} \\
450.030 \pm 0.699\end{array}$ \\
\hline 5 & $\begin{array}{c}\mathrm{a} \\
446.250 \pm 1.675\end{array}$ & $\begin{array}{c}\mathrm{a} \\
472.230 \pm 1.675\end{array}$ & $\begin{array}{c}\mathrm{a} \\
479.180 \pm 1.675\end{array}$ & $\begin{array}{c}\mathrm{a} \\
482.360 \pm 1.675\end{array}$ & $\begin{array}{c}\mathrm{a} \\
509.780 \pm 1.675\end{array}$ & $\begin{array}{c}\mathrm{a} \\
497.640 \pm 1.675\end{array}$ & a $501.870 \pm 1.675$ & $\begin{array}{c}\mathrm{a} \\
527.630 \pm 1.675\end{array}$ \\
\hline
\end{tabular}

Means with in different columns with different superscripts differ significantly $(\mathrm{P}<0.01)$. 
Table(6):The effect of dietary supplementation vitamin C and calcium on feed conversion ratio (FCR)

\begin{tabular}{|c|c|c|c|c|c|c|c|c|}
\hline Treatment & \multicolumn{4}{|c|}{$0.0 \mathrm{mg}$ Vit.C/kg feed } & \multicolumn{4}{|c|}{$150 \mathrm{mg}$ Vit.C/kg feed } \\
\hline & \multicolumn{2}{|c|}{$0.9 \% \mathrm{Ca}$} & \multicolumn{2}{|c|}{$1.1 \% \mathrm{Ca}$} & \multicolumn{2}{|c|}{$0.9 \% \mathrm{Ca}$} & \multicolumn{2}{|c|}{$1.1 \% \mathrm{Ca}$} \\
\hline & Hubbard & Ross & Hubbard & Ross & Hubbard & Ross & Hubbard & Ross \\
\hline Age(week) & Mean \pm SE & Mean \pm SE & Mean \pm SE & Mean \pm SE & Mean \pm SE & Mean \pm SE & Mean \pm SE & Mean \pm SE \\
\hline 1 & $\begin{array}{c}\mathrm{a} \\
1.103 \pm 0.016\end{array}$ & $\begin{array}{c}\mathrm{b} \\
0.982 \pm 0.016\end{array}$ & $\begin{array}{c}\mathrm{b} \\
0.956 \pm 0.016\end{array}$ & $\begin{array}{c}b c \\
0.914 \pm 0.016\end{array}$ & bc $1.019 \pm 0.016$ & $\begin{array}{c}\mathrm{bc} \\
0.936 \pm 0.016\end{array}$ & $\begin{array}{c}\mathrm{bc} \\
0.991 \pm 0.016\end{array}$ & $\begin{array}{c}\mathrm{C} \\
1.917 \pm 0.016\end{array}$ \\
\hline 2 & $\begin{array}{c}\mathrm{A} \\
1.408 \pm 0.008\end{array}$ & $\begin{array}{c}\mathrm{A} \\
1.475 \pm 0.008\end{array}$ & $\begin{array}{c}\mathrm{B} \\
1.459 \pm 0.008\end{array}$ & $\begin{array}{c}\mathrm{A} \\
1.278 \pm 0.008\end{array}$ & A $1.639 \pm 0.008$ & $\begin{array}{c}\mathrm{C} \\
1.524 \pm 0.008\end{array}$ & $\begin{array}{c}\mathrm{C} \\
1.349 \pm 0.008\end{array}$ & $\begin{array}{c}\mathrm{C} \\
1.269 \pm 0.008\end{array}$ \\
\hline 3 & $\begin{array}{c}\mathrm{A} \\
1.347 \pm 0.007\end{array}$ & $\begin{array}{c}\mathrm{A} \\
1.544 \pm 0.007\end{array}$ & $\begin{array}{c}\text { B } \\
1.464 \pm 0.007\end{array}$ & BD $1.422 \pm 0.007$ & A $1.371 \pm 0.007$ & $\begin{array}{c}\mathrm{A} \\
1.532 \pm 0.007\end{array}$ & $\begin{array}{c}\mathrm{C} \\
1.329 \pm 0.007\end{array}$ & $\begin{array}{c}\mathrm{AD} \\
1.614 \pm 0.007\end{array}$ \\
\hline 4 & $\begin{array}{c}\mathrm{A} \\
1.945 \pm 0.015\end{array}$ & $\begin{array}{c}\mathrm{A} \\
1.916 \pm 0.015\end{array}$ & $\begin{array}{c}\text { B } \\
2.006 \pm 0.015\end{array}$ & $\begin{array}{c}\mathrm{A} \\
2.259 \pm 0.015\end{array}$ & B $2.026 \pm 0.015$ & $\begin{array}{c}\mathrm{C} \\
2.394 \pm 0.015\end{array}$ & $\begin{array}{c}\mathrm{D} \\
2.397 \pm 0.015\end{array}$ & $\begin{array}{c}\mathrm{B} \\
2.185 \pm 0.015\end{array}$ \\
\hline 5 & $\begin{array}{c}\mathrm{A} \\
1.659 \pm 0.025\end{array}$ & $\begin{array}{c}\mathrm{A} \\
1.687 \pm 0.025\end{array}$ & $\begin{array}{c}\mathrm{A} \\
2.143 \pm 0.025\end{array}$ & $\begin{array}{c}\mathrm{A} \\
1.958 \pm 0.025\end{array}$ & $\begin{array}{c}\mathrm{AC} \\
2.212 \pm 0.025\end{array}$ & $\begin{array}{c}\mathrm{A} \\
2.879 \pm 0.025\end{array}$ & $\begin{array}{c}C \\
2.712 \pm 0.025\end{array}$ & $\begin{array}{c}\mathrm{B} \\
3.347 \pm 0.025\end{array}$ \\
\hline
\end{tabular}

Means with in different columns with different superscripts differ significantly $(\mathrm{P}<0.01)$. 
Table(7):The effect of dietary supplementation vitamin $\mathrm{C}$ and calcium on mortality rate

\begin{tabular}{|c|c|c|c|c|c|c|c|c|}
\hline \multirow{4}{*}{ Ageatment } & \multicolumn{4}{|c|}{$0.0 \mathrm{mg}$ Vit.C $/ \mathrm{kg}$ feed } & \multicolumn{4}{|c|}{$150 \mathrm{mg}$ Vit.C/kg feed } \\
\hline & \multicolumn{2}{|c|}{$0.9 \% \mathrm{Ca}$} & \multicolumn{2}{|c|}{$1.1 \% \mathrm{Ca}$} & \multicolumn{2}{|c|}{$0.9 \% \mathrm{Ca}$} & \multicolumn{2}{|c|}{$1.1 \% \mathrm{Ca}$} \\
\hline & Hubbard & Ross & Hubbard & Ross & Hubbard & Ross & Hubbard & Ross \\
\hline & Mean \pm SE & Mean \pm SE & Mean \pm SE & Mean \pm SE & Mean \pm SE & Mean \pm SE & Mean \pm SE & Mean \pm SE \\
\hline 1 & $\begin{array}{c}\mathrm{a} \\
0.000 \pm 0.085\end{array}$ & $\begin{array}{c}\mathrm{a} \\
0.000 \pm 0.085\end{array}$ & $\begin{array}{c}\mathrm{a} \\
0.000 \pm 0.085\end{array}$ & $\begin{array}{c}\mathrm{a} \\
0.042 \pm 0.085\end{array}$ & $\begin{array}{c}\mathrm{a} \\
0.000 \pm 0.085\end{array}$ & $\begin{array}{c}\mathrm{a} \\
0.000 \pm 0.085\end{array}$ & $\begin{array}{c}\mathrm{a} \\
0.000 \pm 0.085\end{array}$ & $\begin{array}{c}\mathrm{a} \\
0.000 \pm 0.085\end{array}$ \\
\hline 2 & $\begin{array}{c}\mathrm{a} \\
0.042 \pm 0.067\end{array}$ & $\begin{array}{c}\mathrm{a} \\
0.042 \pm 0.067\end{array}$ & $\begin{array}{c}\mathrm{a} \\
0.042 \pm 0.067\end{array}$ & $\begin{array}{c}\mathrm{a} \\
0.043 \pm 0.067\end{array}$ & $\begin{array}{c}\mathrm{a} \\
0.000 \pm 0.067\end{array}$ & $\begin{array}{c}\mathrm{a} \\
0.000 \pm 0.067\end{array}$ & $\begin{array}{c}\mathrm{a} \\
0.000 \pm 0.067\end{array}$ & $\begin{array}{c}\mathrm{a} \\
0.000 \pm 0.067\end{array}$ \\
\hline 3 & $\begin{array}{c}\mathrm{a} \\
0.000 \pm 0.071\end{array}$ & $\begin{array}{c}\mathrm{a} \\
0.083 \pm 0.071\end{array}$ & $\begin{array}{c}\mathrm{a} \\
0.043 \pm 0.071\end{array}$ & $\begin{array}{c}\mathrm{a} \\
0.000 \pm 0.071\end{array}$ & $\begin{array}{c}\mathrm{a} \\
0.000 \pm 0.071\end{array}$ & $\begin{array}{c}\mathrm{a} \\
0.000 \pm 0.071\end{array}$ & $\begin{array}{c}\mathrm{a} \\
0.042 \pm 0.071\end{array}$ & $\begin{array}{c}\mathrm{a} \\
0.000 \pm 0.071\end{array}$ \\
\hline 4 & $\begin{array}{c}\mathrm{a} \\
0.000 \pm 0.078\end{array}$ & $\begin{array}{c}\mathrm{a} \\
0.000 \pm 0.078\end{array}$ & $\begin{array}{c}\mathrm{a} \\
0.034 \pm 0.078\end{array}$ & $\begin{array}{c}\mathrm{a} \\
0.000 \pm 0.078\end{array}$ & $\begin{array}{c}\mathrm{a} \\
0.000 \pm 0.078\end{array}$ & $\begin{array}{c}\mathrm{a} \\
0.042 \pm 0.078\end{array}$ & $\begin{array}{c}\mathrm{a} \\
0.000 \pm 0.078\end{array}$ & $\begin{array}{c}\mathrm{a} \\
0.000 \pm 0.078\end{array}$ \\
\hline 5 & $\begin{array}{c}\mathrm{a} \\
\pm 0.0000 .000\end{array}$ & $\begin{array}{c}\mathrm{a} \\
0.000 \pm 0.000\end{array}$ & $\begin{array}{c}\mathrm{a} \\
0.000 \pm 0.000\end{array}$ & $\begin{array}{c}\mathrm{a} \\
0.000 \pm 0.000\end{array}$ & $\begin{array}{c}\mathrm{a} \\
0.000 \pm 0.000\end{array}$ & $\begin{array}{c}\mathrm{a} \\
0.000 \pm 0.000\end{array}$ & $\begin{array}{c}\mathrm{a} \\
0.000 \pm 0.000\end{array}$ & $\begin{array}{c}\mathrm{a} \\
0.000 \pm 0.000\end{array}$ \\
\hline Overall mean & $\begin{array}{c}\mathrm{a} \\
0.042 \pm 0.056\end{array}$ & $\begin{array}{c}\mathrm{a} \\
0.083 \pm 0.056\end{array}$ & $\begin{array}{c}\mathrm{a} \\
0.042 \pm 0.056\end{array}$ & $\begin{array}{c}\mathrm{a} \\
0.083 \pm 0.056\end{array}$ & $\begin{array}{c}\mathrm{a} \\
0.000 \pm 0.056\end{array}$ & $\begin{array}{c}\mathrm{a} \\
0.083 \pm 0.056\end{array}$ & $\begin{array}{c}\mathrm{a} \\
0.042 \pm 0.056\end{array}$ & $\begin{array}{c}\mathrm{a} \\
0.000 \pm 0.056\end{array}$ \\
\hline
\end{tabular}

Means with in different columns with different superscripts differ significantly $(\mathrm{P}<0.01)$. 


\section{References}

1. Richards, M.P., 1997. Trace mineral metabolism in the avian embryo. Poult. Sci., 76: 152-164.

2. Wallis, I.R., and Balnave, D.,1984. The influence of environmental temperature, age and sex on the digestibility of amino acid in growing broiler. Br. Poult . Sci., 25:401-407.

3. Kafri,I., and cherry, J.A.1984. Supplemental as corbic acid and heat stress in broiler chicks. Poult.Sci., 63(supp):125.

4. Kutlu, H.R. and Forbes, J.M., 1993. Changes in growth and blood parameters in heat stressed broiler chicks. Live stock prod. Sci., 36:335-350.

5. Orban,J.I., Roland,D.A.Jr., Cummins, K., and Lovell, R.T.,1993. Influence of large doses of ascorbic acid on performance, plasma calcium, bone characteristics and egg shell quality in broiler and leghorn hens. Poult. Sci., 72:691-700.

6. Burley, R.W.,Ewan, A.J and Pearson, J. A.1993.Molecular aspects of synthesis and deposition of hen egg yolk with special reference to low density lipoprotein. Poult .Sci,72:850-855.

7. Keirs,B.,1982.Summer heat loss of fertility in hatching eggs. Poult Digest.,41:352-355.

8. David, E.P and Brake,J.1985. Relationship of dietary ascorbic acid to broiler breeder performance. Poult. Sci., 64: 2041-2048.

9. Dacke, Gc. In : Gc whittow (ed). Sturkies, Avian physiology. 5 th ed. London , UK; Academic press 2000;Pp 472-485.

10. Bains, B.S. 1992. Nutritional approaches to minimize in adequate mineralization proceeding of poultry science symposium at the university of Queen land cotton college, Australia.
11. NRC (National Research council). 1994.Nutrient Requirements of poultry. $9^{\text {th }}$. ed. National Academy press, Washington, DC.

12. Mckee,J.S., and Harrison, P.C.,1995. Effect of supplemental ascorbic acid on the performance of broiler chickens exposed to multiple concurrent stressessor .poult.Sci.,74:1772-1785.

13. Sahin,K., and Kuck, O.,2001. Effect of vitamin $\mathrm{C}$ and vitamin $\mathrm{E}$ on performance, digestion of nutrient and carcass characteristics of Japanese quails reared under chronic heat stress (34c). J.Anim. physiol. Anim. Nutr.,85:335-342.

14. Lohakare, J.D., Ryu,M.H.,Hahn, T.W., Lee, J.K., and chae, B.J.,2005. Effect of supplemental as ascorbic acid on the performance and immunity of commercial broilers. J.Appl.Poult.Sci., 14:1019.

15. Demir,E.,Ozturkcan, O.,Gorgulu, M., Kutlu, H.R., and Okan, F., 1995.(Cited from Ciftic et al 2005). Effect of vitamin $E$ and vitamin $\mathrm{C}$ dietary supplementation on egg production and egg quality of laying hens exposed to chronic heat stress.Revue Med. Vet., 156:107-111.

16. Kassim,H. and Norziha, I 1995.Effect of as corbic acid (vitamin C) supplementation in layer and broiler diets in the tropics.AsianAust .J.Anim.Sci.,8:607-610.

17. Okan,F.,Kutlu,H.R., Canogullari,S., and Baykal,L., 1996. Influence of dietary supplemental ascorbic acid on laying performance of Japanese quails reared under high environmental temperature. Br.poult .Sci., 37: 571-573.

18. Pardue, S. L.; J. P. Thaxton and J. Brake, 1985. Influence of supplemental ascorbic acid on broiler performance following exposure to high environmental 
temperature. Poult Sci., 64:13341338 .

19. Hassan,A.Y.2001.The effect of use some minerals and vitamin $\mathrm{C}$ in diets of broilers under heat stress. Ph.D.Dissert. Coll. Agric and Forestry.Mosul univ.Iraq.

\section{دراسة تأثير إضافة فيتامين C والكالسيوم في بعض صفات الأداء الإنتاجي

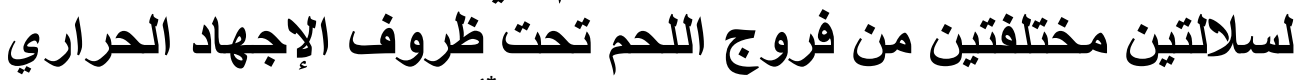

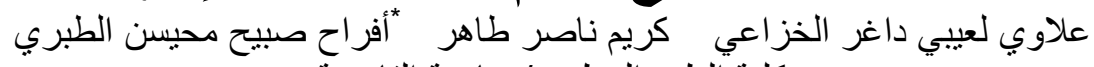 كلية الطب البيطري/ جامعة القادسية الخلاصة}

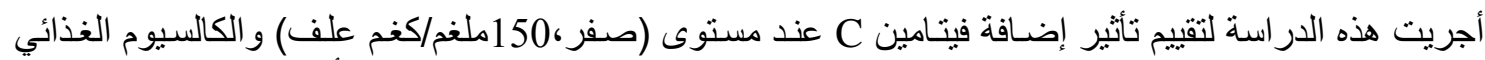

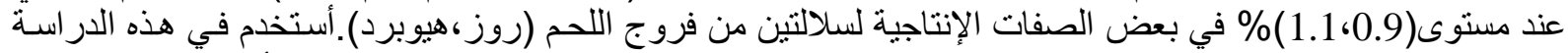

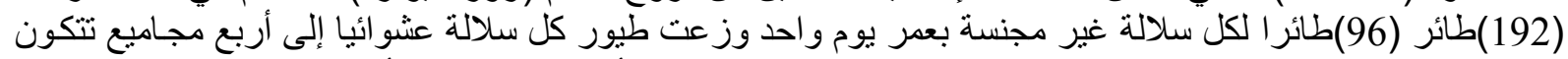

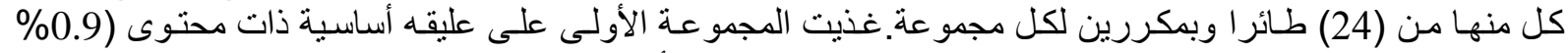

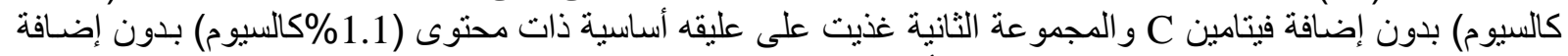

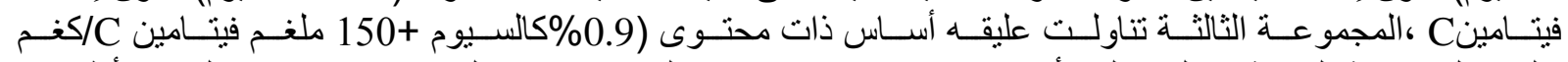

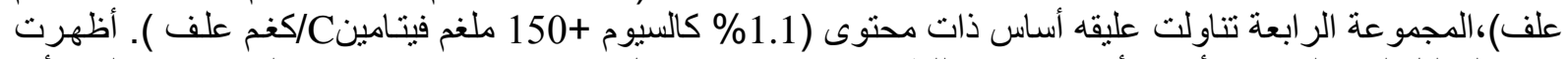

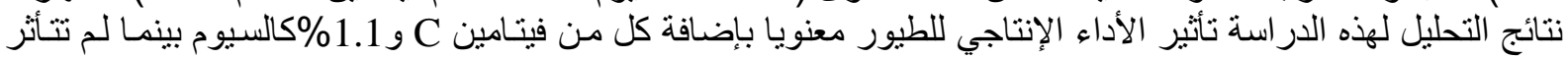
كل من نسبة الهلاكات ونسبة التصافي بإضافة الفيتامين و الكالسيوم للعليقة. 